\title{
Intraventricular Transplantation of Autologous Bone Marrow Mesenchymal Stem Cells via Ommaya Reservoir in Persistent Vegetative State Patients after Haemorrhagic Stroke: Report of Two Cases \& Review of the Literature
}

\author{
Fauzi AA ${ }^{1}$, Suroto NS ${ }^{1}$, Bajamal $\mathrm{AH}^{1}$, Machfoed $\mathrm{MH}^{2}$
}

\begin{abstract}
Background: One of the most devastating diseases, stroke, is a leading cause of death and disability worldwide with severe emotional and economic consequences. The purpose of this article is mainly to report the effect of intraventricular transplantation via an Ommaya reservoir using autologous bone marrow mesenchymal stem cells (BM-MSCs) in haemorrhagic stroke patients.

Case Presentations: Two patients, aged 51 and 52, bearing sequels of haemorrhagic stroke were managed by intraventricular transplantation of BM-MSCs obtained from their own bone marrow. Before the procedure, both patients were bedridden, tracheostomised, on nasogastric (NG) tube feeding and in hemiparesis. The cells were transplanted intraventricularly $\left(20 \mathrm{x} 10^{6}\right.$ cells/2.5 ml) using an Ommaya reservoir, and then repeated transplantations were done after 1 and 2 months consecutively. The safety and efficacy of the procedures were evaluated 3,6 and 12 months after treatment. The National Institute of Health Stroke Scale (NIHSS) was used to evaluate the patients' neurological status before and after treatment. No adverse events derived from the procedures or transplants were observed in the one-year follow-up period, and the neurological status of both patients improved after treatment.

Conclusions: Our report demonstrates that the intraventricular transplantation of BM-MSCs via an Ommaya reservoir is safe and it improves the neurological status of post-haemorrhagic stroke patients. The repeated transplantation procedure is easier and safer to perform via a subcutaneously implanted Ommaya reservoir.
\end{abstract}

Key Words: Haemorrhagic stroke, bone marrow mesenchymal stem cells (BM-MSCs), intraventricular transplantation

\section{Introduction}

Stroke remains a leading cause of morbidity and mortality, with a prevalence rate of about 250-300/100,000 of the total population in Asian countries ${ }^{[1]}$. Approximately $30 \%$ of stroke patients become severely disabled and are left with permanent functional disabilities ${ }^{[2]}$. Early intervention and active rehabilitation still represent the standard therapy for minimizing brain damage and maximizing functional recovery after stroke. However, once functional recovery reaches the plateau phase and any neurological deficits become fixed, patients face severe difficulties, because there is still no known proven treatment ${ }^{[2]}$. Spontaneous intracerebral haemorrhage (ICH) causes $10-20 \%$ of all strokes ${ }^{[3]}$, with outcomes much worse than those of ischemic stroke ${ }^{[4,5]}$. By its nature, ICH causes a variety of neural injury mechanisms: mechanical injury, toxicity, ischemia, inflammation and apoptosis $^{[3]}$. Despite ongoing attempts to find better treatments based on the pathological mechanism of this disease, the outcomes remain poor ${ }^{[6]}$. Nowadays, much attention has been focused on neurorestorative therapies, including stem cell transplantation. Several investigations have reported that direct transplantation or systemic infusion of stem cells results in neurofunctional improvement not only because of the cell replacement mechanism (to replace) but also because of the local host environment being stimulated with various trophic and growth factors secreted by transplanted cells (to stimulate) $)^{[7,8]}$. The route and site of stem cell delivery remain significant issues. Several studies have reported neurofunctional recovery with the intravenous, intracerebral and intraventricular routes of stem cell delivery ${ }^{[2,7,9-12]}$. For example, one study confirmed that all cells reach the target lesions, but more cells are found after intracerebral delivery followed by intraventricular and intravenous delivery ${ }^{[7,13]}$. The indirect route with intravenous injection is non-invasive, but it can create a homing site to other organs far from the target site in the brain ${ }^{[13,14]}$.

Bone marrow mesenchymal stem cells (BM-MSCs) have recently been studied and reported as a potential stem cell source for various central nervous system diseases ${ }^{[5,15,16]}$. Compared with other sources, such as embryonic and other adult stem cells, BM-MSCs are readily accessible, easily expanded in culture and amenable to manipulation ${ }^{[5]}$. The utilization of autologous stem cells would avoid the legal and ethical issues concerning the source of transplant cells in stem cell therapy. BM-MSCs are pluripotent cells that are capable of self-renewal and differentiation in a variety of non-haematological tissues, including brain tissue ${ }^{[5]}$. 


\section{Case Presentations}

This study received legal/ethical clearance from the local medical research ethics committees of Dr. Soetomo General Hospital, Surabaya, Indonesia, following the regulatory guidelines of the country. Moreover, a research permit was obtained from the Research and Development Division of East Java, Indonesia Provincial Government. Informed consent documents, details of the medical treatment and other necessary approval documents were delivered to all patients involved in the study prior to the start of the study. Informed consent was obtained from all the patients included in the study.

Patients in the age-group of 45 and 65 years diagnosed with haemorrhagic stroke within the last 6 months with persistent vegetative state, stable neurological condition in the last 3 months and an initial diagnosis of supratentorial haemorrhagic stroke were recruited for this study. We excluded patients with severe comorbidity, bleeding disorders, history of seizure after stroke, improved neurological condition in the last 3 months, withdrawal from standard therapy after stroke and unwillingness to participate.

\section{Case 1}

The first case was a 51-year-old male patient with a history of left frontoparietal intracerebral haemorrhagic stroke and surgery one year earlier at another hospital. The patient also had a history of hypertension. He had been discharged from the hospital in a persistent vegetative state, and he had been tracheostomised and placed on nasogastric (NG) tube feeding. During the home care, his functional status remained the same, and there was no significant improvement. Then, the family took the patient to our hospital for stem cell treatment. The examination showed the patient was in a persistent vegetative state with a National Institute of Health Stroke Scale (NIHSS) score of 23 and right hemiparesis, and he was tracheostomised and an NG tube was attached. Screening and aspiration of bone marrow stem cells was performed. After 3 weeks, an intraventricular Ommaya reservoir was installed in the right Kocher point, and stem cell transplantation continued intraventricularly. A dose of $20 \times 10^{6}$ in $2.5 \mathrm{cc}$ of normal saline was administered slowly into the right lateral ventricle. Observation during the first 24 hours post-transplantation showed no adverse effects. The patient was sent home in a stable state, and after one and two months, we sequentially transplanted BM-MSCs transcutaneously via the Ommaya reservoir with the same dose. This procedure was done on an outpatient basis. Follow-ups at 3, 6 and 12 months showed improvement in NIHSS scores (20, 19 and 19, respectively). At the last follow-up, the patient was able to sit in a wheelchair, remove the tracheostomy and eat without the NG tube. No other side effects were detected.

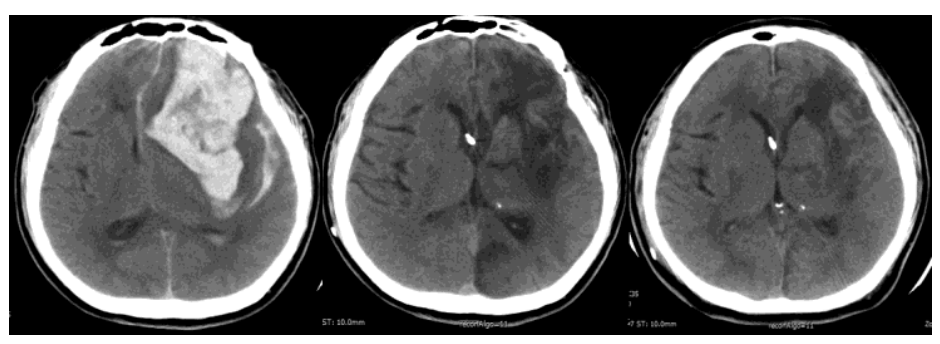

Figure 1: Neuroimaging findings. Changes in occipital infarct area were clearly observed after transplantation, and perihaematomal hypodensity area was reduced after transplantation.
Case 2

The second case was a 52-year-old male patient with a history of left basal ganglia intracerebral haemorrhagic stroke who had undergone an operation approximately 8 months earlier. The patient survived and returned home in a persistent vegetative state with right hemiparesis, and he was tracheostomised and placed on NG tube feeding with an NIHSS score of 17. During his bedridden treatment at home, his functional condition remained the same, and after 3 months, the family created a plan for stem cell therapy. We immediately performed the bone marrow aspiration of BM-MSCs and the expansion of stem cells in our lab. Then, after 3 weeks, we proceeded with the simultaneous cranioplasty operation and transplantation of stem cells via an Ommaya reservoir. Approximately $20 \times 10^{6}$ cells in $2.5 \mathrm{ml}$ of normal saline were injected intraventricularly. Postoperatively, no side effects were found. Then, 1 and 2 months later, we performed a booster transplantation of stem cells with the same dose through the Ommaya reservoir. At observations 3, 6 and 12 months after treatment, the patient showed NIHSS score improvement of 17, 15 and 15, respectively. We cannot provide images for the second case, because the patient's family refused to have a radiological examination after the procedure.

\section{BM-MSC preparation}

The bone marrow of the patient $(10 \mathrm{ml})$ was collected, under local anaesthesia, from the posterior superior iliac crest. The aspirate was mixed and coated with Ficoll and centrifuged at 1,600 rpm for 30 minutes. The 'buffy coat' located on Ficoll-PBS was collected, and cells were then inoculated on a $10-\mathrm{cm}^{2}$ plate. Then, cell incubation was performed at $37^{\circ} \mathrm{C}$ with humidity of $5 \% \mathrm{CO}_{2}$ for 24 hours. Microscopic evaluation was done every day, and every 3 days, the cells were washed with $10 \mathrm{ml}$ PBS and $10 \mathrm{ml} \mathrm{CCM}$ was added until the cells became confluent between $70-90 \%$. Thus, cells were cultured and expanded to reach the target amount within 21-28 days and were used for intraventricular transplantation. At each procedure, cells were fully dispersed and dissolved in $2.5 \mathrm{ml}$ normal saline and injected intraventricularly in 5-10 minutes using a 5-ml syringe.

\section{Discussion}

Some in vivo and in vitro studies have been conducted to assess the effectiveness of stem cell transplantation therapy in stroke ${ }^{[13,17]}$. The results have shown that stem cell therapy in stroke is safe, but they have not been optimal. Clinical research on the effects of stem cell therapy in haemorrhagic stroke has been reported ${ }^{[11,12]}$. Purandare et al. reported that the intrathecal administration of autologous bone marrow stromal cells (BMSCs) and allogeneic cord blood stem cells (CBSCs) in patients aged 54 years with haemorrhagic stroke produced functional and neurological improvements based on a comparison of NIHSS scores before and after treatment ${ }^{[11]}$. At the two-year follow-up, patients showed a significant improvement of motor, sensory-cognitive and speech function ${ }^{[11]}$. Sharma et al. reported that the intrathecal administration of autologous BMSC therapy as part of rehabilitation therapy produced an improvement of neurological condition (cognitive and motor strength) without side effects ${ }^{[12]}$.

A wide variety of stem cell therapies in stroke patients have been performed. For example, they varied in terms of the time of administration, route of administration, dosage and source of stem cells and administration of adjuvant therapy to improve the effectiveness of the transplantation ${ }^{[13]}$. Unfortunately, there have been no significant achievements, and research still goes on, both in experimental animals and humans ${ }^{[13,17]}$. Stem cell therapy with indirect techniques, such as intra-arterial and intravenous techniques, has been performed with variable results ${ }^{[1,17,18]}$. Theoretically, it is 
said that the existence of the blood-brain barrier makes indirect or systemic transplantation ineffective at repairing the abnormalities in the brain ${ }^{[13]}$. Direct intracranial stem cell transplantation is another option for a more effective homing process to the brain ${ }^{[13]}$. Direct intraparenchymal stem cell transplantation has been widely reported in the case of stroke infarction ${ }^{[2,14]}$. Direct intraventricular stem cell transplantation techniques have only been reported in the case of amyotrophic lateral sclerosis (ALS) and ischemic encephalopathy in paediatric patients ${ }^{[9,10,19]}$. In contrast to Parkinson's disease, for which stem cell therapy has been shown to be quite effective, stroke destroys the highly complicated architecture of the brain tissue and is often accompanied by vascularization defects and chronic systemic disease $^{[16]}$. In haemorrhagic stroke, brain tissue damage is more severe (often fatal), and more serious neurological deficits are observed $^{[4,6]}$

The ventricular system, which is divided into four sections (two lateral ventricles, ventricle 3 , and ventricle 4$)^{[20]}$, contains a thin wall composed of ependymal cells ${ }^{[20,21]}$. The permeable ependymal cell layer is quite receptive to certain medications, including stem cell therapy, to the brain parenchyma ${ }^{[21,22]}$. In the lateral ventricle, the ventricular wall is surrounded by a neurogenic area known as the subventricular zone $(\mathrm{SVZ})^{[22-25]}$ that continuously produces new neurons ${ }^{[22,23,25-27]}$. The SVZ was first discovered in mice, then in larger mammals and later, it was found to be present in humans ${ }^{[24,27]}$. The fact that the neurogenic niche area is very close to the lateral ventricle explains why the intraventricular administration of stem cells is an effective route for stem cell therapy in stroke cases ${ }^{[9]}$. This is because the lateral ventricles are an easy target, and of course, they directly stimulate the $\mathrm{SVZ}^{[9,22]}$.

The natural ventricular system and its cerebrospinal fluid activities regulate the endogenous stimulation of neuronal differentiation in the neural regeneration process, where the plexus choroideus produces certain substances in the brain during development or neural regeneration after brain injury ${ }^{[20,21]}$. The occurrence of endogenous neurogenesis has been widely reported in several studies ${ }^{[20,22-24,26-27]}$.

In this study, we used BM-MSCs from the patients' autologous BMMSCs, thus avoiding the ethical issues and preventing complications related to stem cell source. The likelihood of rejection by the body was minimal, and there was no need to use immunosuppressant drugs. Seizure complications are usually due to the effects of foreign bodies and mass effects ${ }^{[8]}$. This was avoided, because in this study, the transplants were given intraventricularly without causing a permanent mass effect or irritating effect in the brain tissue.

Many experimental studies on the use of adult mesenchymal stem cells (MSCs) have been carried out on a wide variety of diseases $^{[5,15,16]}$. It is well known that the transplantation of MSCs taken from the bone marrow can not only affect the tissue repair process by stimulating the processes of proliferation, migration and differentiation of progenitor cells endogenous in all the tissues of the body, but also suppress inflammatory and immune reactions and prevent apoptosis ${ }^{[15,28]}$.

Stem cell transplantation dose has also varied greatly in previous studies $^{[1,2,9,10,12,18]}$ and there is currently no standard dose for stem cell therapy associated with administration route and disease type ${ }^{[28]}$. Wang et al.'s research comparing stem cell doses showed that an optimal dose for therapeutic stem cell transplantation is necessary, but this does not mean that a larger dose of stem cells is better. For example, for intraparenchymal sites, overly large transplant doses can affect the nutrition of grafted cells, and when administered intravascularly, they could cause microemboli and the occlusion of blood vessels ${ }^{[28]}$.
In this study, we performed stem cell transplantation intraventricularly with doses of $20 \times 10^{6}$, higher than those in previous studies. Unlike in the intraparenchymal transplantation technique, in direct intraventricular transplantation, dosage adjustment is more flexible, because it can be controlled by reducing the ventricular fluid as needed while the transplantation procedure is performed. The risk of increased intracranial pressure and mass effect can be avoided. In this study, we used a dose of $20 \times 10^{6} \mathrm{BM}$ MSCs administered in $2.5 \mathrm{ml}$ of liquid to avoid hyper-concentration as well as excess volume, and we found no complications regarding increased intracranial pressure or other complications, such as infection or seizure.

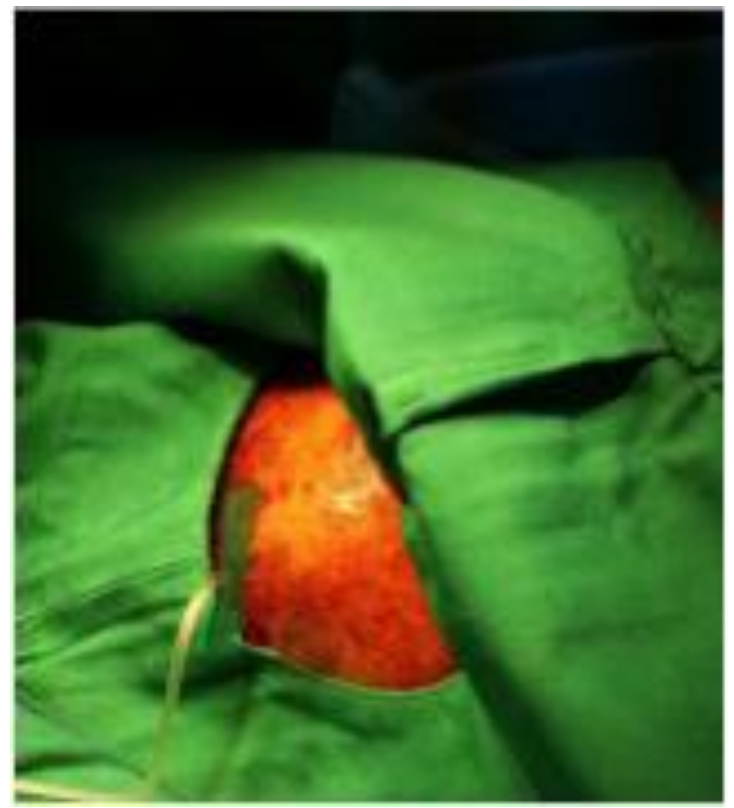

Figure 2: Booster transplantation via Ommaya reservoir

The intraventricular transplantation of stem cells in stroke patients has not been reported widely. The three previous reports were case reports (i.e. two cases of children with post-hypoxic encephalopathy and one case with ALS). Jozwiak et al. performed intraventricular transplantation using autologous umbilical cord stem cells in a 16month-old child with post-hypoxic encephalopathy with fairly good results ${ }^{[9]}$. Luan et al. reported a case of an infant with severe ischemic encephalopathy that was successfully treated with human foetal neural stem cell transplantation into the cerebral ventricle with no adverse effects ${ }^{[10]}$. Baek et al. conducted a transplantation technique using an Ommaya reservoir in a case of ALS. The Ommaya reservoir facilitated any additional injections (booster therapy ${ }^{[19]}$.

\section{Conclusion}

We have reported two cases of persistent vegetative state patients after haemorrhagic stroke treated with the intraventricular transplantation of BM-MSCs via an Ommaya reservoir. Evaluation and follow-up of patients for up to 12 months showed that this treatment is a safe and well-tolerated procedure. Both patients experienced improvement of functional status post-transplantation, as indicated by their NIHSS scores. To the best of our knowledge, this is the first report of the intraventricular transplantation of autologous BM-MSCs using Ommaya reservoirs for stroke and it may be developed for future therapies. Further large clinical studies are needed to determine the efficacy of this procedure for treating disability after stroke. 


\section{References}

1. Bhasin A, Srivastava MVP, Kumaran SS, Mohanty S, Bhatia R, Garg A, Airan B. Autologous mesenchymal stem cells in chronic stroke. Cerebrovascular Diseases Extra. 2011;1(1):93-104.

2. Kondziolka D, Steinberg GK, Wechsler L, Meltzer CC, Elder E, Gebel J, Decesare S, Jovin T, Zafonte R, Lebowitz J, Flickinger JC, Tong D, Marks MP, Jamieson C, Luu D, Bell-Stephens T, Teraoka J. Neurotransplantation for patients with subcortical motor stroke: a phase 2 randomized trial. J Neurosurg. 2005;103(1):38-45.

3. Seyfried D, Ding J, Han Y, Li Y, Chen J, Chopp M. Effect of intravenous administration of human bone marrow stromal cells after intracerebral hemorrhage in rats. J Neurosurg. 2006;104(2):313-8.

4. Keep RF, Hua Y, Xi G. Intracerebral haemorrhage: mechanisms of injury and therapeutic targets. Lancet Neurol. 2012;11(8):720-31.

5. Seyfried DM, Han Y, Yang D, Ding J, Shen LH, Savant-Bhonsale $\mathrm{S}$, Chopp M. Localization of bone marrow stromal cells to the injury site after intracerebral hemorrhage in rats. J Neurosurg. 2010;112(2):329-35.

6. Rincon F, Mayer SA. Clinical review: Critical care management of spontaneous intracerebral hemorrhage. Crit Care. 2008;12(6):237.

7. Guzman R, Choi R, Gera A, De Los Angeles A, Andres RH, Steinberg GK. Intravascular cell replacement therapy for stroke. Neurosurg Focus. 2008;24(3-4):E15.

8. Hammer MD, Kondziolka D, Wechsler LR. Safety and Efficacy of Transplanting Immortalized Neural Stem Cells in Stroke Patients. In: Savitz SI, Rosenbaum DM, editors. Stroke Recovery with Cellular Therapies. New Jersey: Humana Press; 2008. p. 1-9.

9. Jozwiak S, Habich A, Kotulska K, Sarnowska A, Kropiwnicki T, Janowski M, Jurkiewicz E, Lukomska B, Kmiec T, Walecki J, Roszkowski M, Litwin M, Oldak T, Boruczkowski D, DomanskaJanik K. Intracerebroventricular Transplantation of Cord BloodDerived Neural Progenitors in a Child With Severe Global Brain Ischemic Injury. Cell Med. 2010;1(2):71-80.

10. Luan Z, Yin GC, Hu XH, Qu SQ, Wu NH, Yan FQ, Qian YM, Jin HY, Gong XJ. Treatment of an infant with severe neonatal hypoxic-ischemic encephalopathy sequelae with transplantation of human neural stem cells into cerebral ventricle. Zhonghua Er Ke Za Zhi. 2005; 43(8):580-3;

11. Purandare C, Belle V, Shitole DG, Joshi M. Stem Cell Therapy for Hemorrhagic Stroke: A Single Case Study Report. Journal of Neuroscience. 2012;2(2):22-6.

12. Sharma A, Sane H, Badhe P, Kulkarni P, Chopra G, Lohia M, Gokulchandran N. Autologous Bone Marrow Stem Cell Therapy Shows Functional Improvement in Hemorrhagic Stroke. Indian Journal of Clinical Practice. 2012;23(2):100-5.

13. Bliss T, Guzman R, Daadi M, Steinberg GK. Cell transplantation therapy for stroke. Stroke. 2007;38(part 2):817-26.

14. Willing AE, Shahaduzzaman MD. Delivery Routes for Cell Therapy in Stroke. In: Jolkkonen J, Walczak P, editors. Cell-Based Therapies in Stroke. Wien: Springer; 2013. p. 15-28.

15. Calio ML, Marinho DS, Ko GM, Ribeiro RR, Carbonel AF, Oyama LM, Ormanji M, Guirao TP, Calio PL, Reis LA, Simoes
Mde J, Lisboa-Nascimento T, Ferreira AT, Bertoncini CR Transplantation of bone marrow mesenchymal stem cells decreases oxidative stress, apoptosis, and hippocampal damage in brain of a spontaneous stroke model. Free Radic Biol Med. 2014;70:141-54

16. Chopp M, Li Y, Shen L. Transplantation of bone marrow stromal cells for the treatment of stroke. In: Savitz SI, Rosenbaum DM, editors. Stroke Recovery with Cellular Therapies. New Jersey: Humana Press; 2008. p. 11-27.

17. Srivastava P. Restorative therapy in stroke using stem cells. Neurol India. 2009;57(4):381-6.

18. Prasad K, Sharma A, Garg A, Mohanty S, Bhatnagar S, Johri S, Singh KK, Nair V, Sarkar RS, Gorthi SP, Hassan KM, Prabhakar S, Marwaha N, Khandelwal N, Misra UK, Kalita J, Nityanand S. Intravenous autologous bone marrow mononuclear stem cell therapy for ischemic stroke: a multicentric, randomized trial. Stroke. 2014 ;45(12):3618-24.

19. Baek W, Kim YS, Koh SH, Lim SW, Kim HY, Yi HJ, Kim H. Stem cell transplantation into the intraventricular space via an Ommaya reservoir in a patient with amyotrophic lateral sclerosis. J Neurosurg Sci. 2012;56(3):261-3.

20. Lowery LA, Sive H. Totally Tubular: The Mystery behind Function and Origin of the Brain Ventricular System. Bioessays. 2009;31(4):446-58.

21. Falcao AM, Marques F, Novais A, Sousa N, Palha JA, Sousa JC. The path from the choroid plexus to the subventricular zone: gone with the flow!. Front Cell Neurosci. 2012;6:34.

22. Kazanis I. The subependymal zone neurogenic niche: a beating heart in the centre of the brain: how plastic is adult neurogenesis? Opportunities for therapy and questions to be addressed. Brain. 2009;132(Pt 11):2909-21.

23. Gage FH. Molecular and cellular mechanisms contributing to the regulation, proliferation and differentiation of neural stem cells in the adult dentate gyrus. The Keio Journal of Medicine. 2010;59(3):79-83.

24. Kazanis I. Can Adult Neural Stem Cells Create New Brains? Plasticity in the Adult Mammalian Neurogenic Niches: Realities and Expectations in the Era of Regenerative Biology. Neuroscientist. 2012;18(1): 15-27.

25. Okano H. Neural stem cells: progression of basic research and perspective for clinical application. Keio J Med. 2002;51(3):11528. Bordey A.

26. Adult neurogenesis: Basic concepts of signaling. Cell Cycle. 2006; 5(7):722-8.

27. Doetsch F, Caille I, Lim DA, Garcia-Verdugo JM, Alvarez-Buylla A. Subventricular zone astrocytes are neural stem cells in the adult mammalian brain. Cell. 1999;97(6):703-16.

28. Wang LQ, Lin ZZ, Zhang HX, Shao B, Xiao L, Jiang HG, Zhuge QC, Xie LK, Wang B, Su DM, Jin KL. Timing and dose regimens of marrow mesenchymal stem cell transplantation affect the outcomes and neuroinflammatory response after ischemic stroke. CNS Neurosci Ther. 2014;20(4):317-26. 


$\begin{array}{ll}\text { Abbreviations } & \\ \text { ALS } & \text { : Amyotrophic Lateral Sclerosis } \\ \text { BM-MSCs } & \text { : Bone Marrow Mesenchymal Stem Cells } \\ \text { BMSCs } & \text { : Bone Marrow Stromal Cells } \\ \text { CBSCs } & \text { : Cord Blood Stem Cells } \\ \text { CCM } & \text { : Complete Culture Medium } \\ \text { ICH } & \text { : Intracerebral Haemorrhage } \\ \text { MSCs } & \text { : Mesenchymal Stem Cells } \\ \text { NG } & \text { : Nasogastric } \\ \text { NIHSS } & \text { : National Institute of Health Stroke Scale } \\ \text { PBS } & \text { : Phosphate-buffered Saline } \\ \text { SVZ } & : \text { Subventricular Zone }\end{array}$

Potential Conflicts of Interests

None

\section{Informed Consent and Ethical Approvals}

The study was conducted with the human subject's understanding and consent. The Ethical Committee at Dr. Soetomo General Hospital approved this study.

\section{Acknowledgments}

The Authors would like to acknowledge the Stem Cell and Regenerative Medicine Center at Dr. Soetomo General Hospital and Surabaya Neuroscience Intitute for its support in this study.

\section{Corresponding Author}

Asra Al Fauzi, Associate Professor of Clinical Neurological Surgery, Chief of Neurovascular and Neuroendovascular Therapy Center, Department of Neurosurgery, Airlangga University School of Medicine, Dr. Soetomo General Hospital - Diagnostic Center Building, Surabaya Neuroscience Institute, J1. Prof. Dr. Moestopo 6-8, Surabaya 60285 - Indonesia, Email: asrafauzi@ yahoo.com 